PROCEEDINGS OF THE

AMERICAN MATHEMATICAL SOCIETY

Volume 132, Number 4, Pages 1005-1011

S 0002-9939(03)07285-X

Article electronically published on November 19, 2003

\title{
STRONG COMPARISON PRINCIPLE FOR SOLUTIONS OF QUASILINEAR EQUATIONS
}

\author{
M. LUCIA AND S. PRASHANTH
}

(Communicated by David S. Tartakoff)

\begin{abstract}
Let $\Omega \subset \mathbb{R}^{N}, N \geq 1$, be a bounded smooth connected open set and $\mathbf{a}: \Omega \times \mathbb{R}^{N} \rightarrow \mathbb{R}^{N}$ be a map satisfying the hypotheses (H1)-(H4) below. Let $f_{1}, f_{2} \in \mathrm{L}_{l o c}^{1}(\Omega)$ with $f_{2} \geq f_{1}, f_{1} \not \equiv f_{2}$ in $\Omega$ and $u_{1}, u_{2} \in \mathcal{C}^{1, \theta}(\bar{\Omega})$ with $\theta \in(0,1]$ be two weak solutions of

$$
\left(P_{i}\right) \quad-\operatorname{div}\left(\mathbf{a}\left(x, \nabla u_{i}\right)\right)=f_{i} \quad \text { in } \Omega, \quad i=1,2 .
$$

Suppose that $u_{2} \geq u_{1}$ in $\Omega$. Then we show that $u_{2}>u_{1}$ in $\Omega$ under the following assumptions: either $u_{2}>u_{1}$ on $\partial \Omega$, or $u_{1}=u_{2}=0$ on $\partial \Omega$ and $u_{1} \geq 0$ in $\Omega$. We also show a measure-theoretic version of the Strong Comparison Principle.
\end{abstract}

\section{INTRODUCTION}

Let $\Omega \subset \mathbb{R}^{N}, N \geq 1$, be a bounded, connected open set and consider a map $\mathbf{a}=\left(a_{1}, \ldots, a_{N}\right): \Omega \times \mathbb{R}^{N} \rightarrow \mathbb{R}^{N}$ satisfying the following hypotheses (H1)-(H4):

There exist constants $\mathcal{K} \in[0,1], \gamma, \Gamma \in(0, \infty), p \in(1, \infty)$ such that:

(H1) $a_{i}(x, 0)=0 \forall x \in \Omega, a_{i} \in \mathcal{C}\left(\Omega \times \mathbb{R}^{N}\right) \cap \mathcal{C}^{1}\left(\Omega \times \mathbb{R}^{N} \backslash\{0\}\right), i=1, \ldots, N$;

(H2) $\sum_{i, j=1}^{N} \frac{\partial a_{i}}{\partial \eta_{j}}(x, \eta) \xi_{i} \xi_{j} \geq \gamma(\mathcal{K}+|\eta|)^{p-2}|\xi|^{2}, \quad \forall(x, \eta, \xi) \in \Omega \times \mathbb{R}^{N} \backslash\{0\} \times \mathbb{R}^{N} ;$

(H3) $\sum_{i, j=1}^{N}\left|\frac{\partial a_{i}}{\partial \eta_{j}}(x, \eta)\right| \leq \Gamma(\mathcal{K}+|\eta|)^{p-2}, \quad \forall(x, \eta) \in \Omega \times \mathbb{R}^{N} \backslash\{0\} ;$

(H4) $\sum_{i, j=1}^{N}\left|\frac{\partial a_{i}}{\partial x_{j}}(x, \eta)\right| \leq \Gamma(\mathcal{K}+|\eta|)^{p-2}|\eta|, \quad \forall(x, \eta) \in \Omega \times \mathbb{R}^{N} \backslash\{0\}$.

As an easy consequence of hypotheses (H1)-(H4) we obtain that $\mathbf{a}(x, \cdot)$ is a strictly monotone vector field on $\mathbb{R}^{N}$. This motivates the following hypothesis:

(H5) $a_{i} \in \mathcal{C}\left(\Omega \times \mathbb{R}^{N}\right), i=1, \ldots, N$, and

$$
\begin{array}{lll}
\left\langle\mathbf{a}\left(x, \eta_{1}\right)-\mathbf{a}\left(x, \eta_{2}\right), \eta_{1}-\eta_{2}\right\rangle \geq 0 & \forall \eta_{1}, \eta_{2} \in \mathbb{R}^{N}, & \forall x \in \Omega, \\
\left\langle\mathbf{a}\left(x, \eta_{1}\right)-\mathbf{a}\left(x, \eta_{2}\right), \eta_{1}-\eta_{2}\right\rangle=0 \quad \text { iff } \eta_{1}=\eta_{2}, & \forall x \in \Omega .
\end{array}
$$

A well-known example of a vector field satisfying hypotheses (H1)-(H4) is given by $\mathbf{a}(\eta)=|\eta|^{p-2} \eta, p \in(1, \infty)$.

Received by the editors August 20, 2002.

2000 Mathematics Subject Classification. Primary 35B50.

The first author was supported by Swiss National Foundation, Contract No. 8220-64676.

The second author was supported by Indo-French Project (IFCPAR) No. 1901-2. 
Consider two functions $f_{1}, f_{2}$ satisfying the following hypothesis:

(F) $f_{1}, f_{2} \in \mathrm{L}_{l o c}^{1}(\Omega), f_{2} \geq f_{1}, f_{1} \not \equiv f_{2}$ in $\Omega$.

We are interested in the following question:

Let a satisfy some of the hypotheses in (H1)-(H5) and $f_{1}, f_{2}$ satisfy the hypothesis $(\mathrm{F})$. Let $u_{1}, u_{2} \in \mathcal{C}^{1, \theta}(\bar{\Omega})$ for some $\theta \in(0,1]$ be two weak solutions of

$$
\left(P_{i}\right) \quad-\operatorname{div}\left(\mathbf{a}\left(x, \nabla u_{i}\right)\right)=f_{i} \quad \text { in } \Omega, \quad i=1,2 .
$$

Suppose $u_{2} \geq u_{1}$ in $\Omega$. Then, is it true that $u_{2}>u_{1}$ in $\Omega$ ?

If the answer to the above question is "yes" we say that the Strong Comparison Principle, (SCP) for short, holds for the problem $\left(P_{i}\right)$.

The first important result in this direction was given by [3]. More precisely, they show that (SCP) holds under either one of the following assumptions:

$(\tilde{A} 1) u_{2}>u_{1}$ on $\partial \Omega$,

$(\tilde{A} 2) u_{1}=u_{2}=0$ on $\partial \Omega, u_{2} \geq u_{1} \geq 0$ in $\Omega$ and interior $\left\{x \in \Omega: f_{1}(x)=f_{2}(x)\right\}$ is an empty set.

Later, in a nice work, Cuesta and Takáč [1] have contributed towards weakening the assumption $(\tilde{A} 2)$, while assuming that $\partial \Omega$ has only one component. Recently, [4] and [5] have shown that (SCP) holds without any other restrictions if we are in the radial case. But, the full (SCP) still remains open.

In this work, we show that (SCP) holds with an assumption similar to $(\tilde{A} 1)$ or an assumption like: $u_{1}=u_{2}=0$ on $\partial \Omega, u_{2} \geq u_{1} \geq 0$ in $\Omega$. In the general case, when we have only $u_{2} \geq u_{1}$ in $\Omega$, we show that the $N$-dimensional Hausdorff measure of the coincidence set $\left\{x \in \Omega: u_{1}(x)=u_{2}(x)\right\}$ is zero.

The proof we give will be based on the divergence theorem applied to a particular vector field $\mathbf{G}$ generated by the solutions of $\left(P_{i}\right)$. In the articles [3] and [1], the proofs are based more on PDE techniques such as the maximum principle. We now state the theorems that we will prove.

The first theorem shows a measure-theoretic version of (SCP) without any restrictions on the boundary data.

Theorem 1.1. Let a satisfy the hypothesis (H5) and $f_{1}, f_{2}$ the hypothesis (F). Let $u_{i} \in \mathcal{C}^{1, \theta}(\bar{\Omega}), \theta \in(0,1]$ be a weak solution of $\left(P_{i}\right), i=1,2$ with $u_{2} \geq u_{1}$ in $\Omega$. Then the $N$-dimensional Hausdorff measure of the set

$$
\left\{x \in \Omega: u_{1}(x)=u_{2}(x)\right\} \cap\left\{x \in \Omega: f_{1}(x) \neq f_{2}(x)\right\}
$$

is zero.

The second theorem is a generalised version of the corresponding results in 6 ] and 3 for "elliptic" vector fields a satisfying hypotheses (H1)-(H4). We remark that we need only the monotonicity hypothesis (H5) on the map a.

Theorem 1.2. Let a satisfy the hypothesis (H5), $f_{1}, f_{2} \in \mathrm{L}_{l o c}^{1}(\Omega)$ with $f_{2} \geq f_{1}$ in $\Omega$ and $u_{i} \in \mathcal{C}^{1, \theta}(\bar{\Omega}), \theta \in(0,1]$ be a weak solution of $\left(P_{i}\right), i=1,2$. Then, $\inf _{\Omega}\left(u_{2}-u_{1}\right) \geq \inf _{\partial \Omega}\left(u_{2}-u_{1}\right)$.

The last theorem shows (SCP) for nonnegative solutions of $\left(P_{i}\right)$ with zero Dirichlet boundary conditions. Here we remove the restriction imposed in [1] that $\partial \Omega$ has only one connected component.

Theorem 1.3. Assume that $\partial \Omega$ is a $\mathcal{C}^{2}$-manifold and that a satisfies hypotheses (H1)-(H4). Let $f_{1}, f_{2} \in L^{\infty}(\Omega)$ be two functions satisfying the hypothesis (F) and 
$u_{i} \in \mathcal{C}^{1, \theta}(\bar{\Omega}), \theta \in(0,1]$ be weak solutions of $\left(P_{i}\right)(i=1,2)$. Assume that $u_{1}=u_{2}=$ 0 on $\partial \Omega, u_{2} \geq u_{1} \geq 0$ in $\Omega$. Then, (SCP) holds in $\Omega$, i.e., $u_{2}>u_{1}$ in $\Omega$.

\section{Preliminary Results}

Given $U \subset \Omega$ a set of finite perimeter in $\Omega$, we denote by $\partial_{\star} U$ the measuretheoretic boundary of $U$ in $\Omega$ (see [2] for more details). The proof of the above theorems depends on the following version of the divergence theorem:

Proposition 2.1. Let $\mathbf{G}: \Omega \rightarrow \mathbb{R}^{N}$ be a continuous vector field, $U \subset \subset \Omega$ be an open set with finite perimeter in $\Omega$. If, in the sense of distributions,

$$
-\operatorname{div} \mathbf{G}=f \in \mathrm{L}^{1}(U),
$$

then the following version of the divergence theorem is true:

$$
-\int_{\partial_{\star} U}\langle\mathbf{G}, \nu\rangle d \sigma=\int_{U} f
$$

where $\partial_{\star} U$ is the measure-theoretic boundary of $U$ in $\Omega, \sigma=\mathcal{H}^{N-1}$ is the $(N-1)$ dimensional Hausdorff measure in $R^{N}$, and $\nu$ is the unique measure-theoretic unit outer normal to the boundary of $U$ that exists $\mathcal{H}^{N-1}$ a.e. on $\partial_{\star} U$.

Proof. We write $\mathbf{G}=\left(G_{1}, \ldots, G_{N}\right)$. Let $\rho_{\epsilon} \in \mathcal{C}_{0}^{\infty}(\Omega)$ be the standard family of mollifier functions. We approximate $\mathbf{G}$ by the following smooth vector fields: $\mathbf{G}_{\epsilon}=$ $\left(G_{1} * \rho_{\epsilon}, \ldots, G_{N} * \rho_{\epsilon}\right)$. It is easy to check that

$$
\mathbf{G}_{\epsilon} \rightarrow \mathbf{G} \text { in } \mathcal{C}_{\text {loc }}\left(\Omega ; \mathbb{R}^{N}\right), \quad \operatorname{div}\left(\mathbf{G}_{\epsilon}\right) \rightarrow \operatorname{div} \mathbf{G} \text { in } \mathrm{L}_{l o c}^{1}(\Omega) .
$$

For every $\mathbf{G}_{\epsilon}$ the divergence theorem in equation (2.2) is true (see [2], Thm. 1, p. 209). The proposition now follows from the approximating property of $\mathbf{G}_{\epsilon}$ to G.

Given $u_{1}, u_{2} \in \mathcal{C}^{1}(\Omega)$, we set for each $\epsilon \in \mathbb{R}$,

$$
L_{\epsilon}=\left\{x \in \Omega:\left(u_{2}-u_{1}\right)(x)<\epsilon\right\},
$$

and define the following vector field:

$$
\mathbf{G}: \Omega \rightarrow \mathbb{R}^{N}, \quad \mathbf{G}(x)=\mathbf{a}\left(x, \nabla u_{2}(x)\right)-\mathbf{a}\left(x, \nabla u_{1}(x)\right) .
$$

We first recall the following standard result in geometric measure theory; see, for example, Theorem 1, Section 5.5 of [2].

Lemma 2.2. For $\mathcal{H}^{1}$ a.e. $\epsilon \in \mathbb{R}$, the set $L_{\epsilon}$ is of finite perimeter in $\Omega$.

We note the following important property.

Proposition 2.3. Let $u_{1}, u_{2} \in \mathcal{C}^{1}(\Omega)$ and consider $L_{\epsilon}$, $\mathbf{G}$ defined by (2.3), (2.4). Assume $L_{\epsilon}$ to be of finite perimeter in $\Omega$ and that a satisfies (H5). Then, $\langle\mathbf{G}, \nu\rangle(x) \geq 0$ for all $x \in \partial_{\star} L_{\epsilon} \cap \Omega$ (here $\nu$ is the measure-theoretic unit outer normal).

Proof. Let $x_{0} \in \partial_{\star} L_{\epsilon} \cap \Omega$ be such that $\left|\mathbf{G}\left(x_{0}\right)\right| \neq 0$. Let us set $w=u_{2}-u_{1}$. By (H5), $\nabla w\left(x_{0}\right) \neq 0$. Therefore, $\partial L_{\epsilon}$ is a $\mathcal{C}^{1}$-manifold in a neighborhood of $x_{0}$, and hence the measure-theoretic unit outer normal is explicitly given in this neighborhood by $\nu(x)=\frac{\nabla w(x)}{|\nabla w(x)|}$. The proof of the proposition now follows from the definition of $\mathbf{G}$ and (H5). 
Lemma 2.4. Assume that a satisfies (H5). Let $U \subset \mathbb{R}^{N}$ be an open set. Let $w_{1}, w_{2} \in \mathcal{C}^{1, \theta}(\bar{U}), \theta \in(0,1]$, be two weak solutions of the problem:

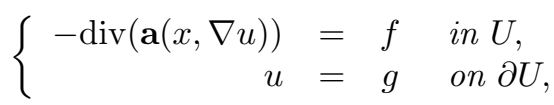

where $f \in \mathrm{L}_{\text {loc }}^{1}(U)$ and $g \in \mathcal{C}(\partial U)$. Then, $w_{1} \equiv w_{2}$ in $U$.

Proof. We note that $w_{2}-w_{1} \in \mathcal{C}^{1}(U)$ with $w_{2}-w_{1}=0$ on $\partial U$. Hence, by the definition of weak solutions,

$$
\int_{U}\left\langle\mathbf{a}\left(x, \nabla w_{2}\right)-\mathbf{a}\left(x, \nabla w_{1}\right), \nabla\left(w_{2}-w_{1}\right)\right\rangle=0 .
$$

Since $\left\langle\mathbf{a}\left(x, \nabla w_{2}\right)-\mathbf{a}\left(x, \nabla w_{1}\right), \nabla\left(w_{2}-w_{1}\right)\right\rangle \geq 0$, we conclude that $\left\langle\mathbf{a}\left(x, \nabla w_{2}\right)-\right.$ $\left.\mathbf{a}\left(x, \nabla w_{1}\right), \nabla\left(w_{2}-w_{1}\right)\right\rangle \equiv 0$ in $U$ and hence $\nabla\left(w_{2}-w_{1}\right) \equiv 0$ (by (H5)). This proves the lemma.

\section{SCP FOR MONOTONE VECTOR FIELDS}

In this section we consider a vector field a that satisfies only (H5). Under this mild assumption, we first show that the measure of the set where the (SCP) possibly fails to hold in $\left\{x \in \Omega: f_{2}(x)>f_{1}(x)\right\}$ has to be of measure zero:

Proof of Theorem 1.1. Let $Z=\left\{x \in \Omega: u_{1}(x)=u_{2}(x)\right\}$ and $P_{f}=\{x \in \Omega$ : $\left.f_{1}(x) \neq f_{2}(x)\right\}$. Clearly, it is enough to show that $\mathcal{H}^{N}\left(Z \cap P_{f} \cap B\right)=0$ for any ball $B \subset \subset \Omega$. Fix any such ball $B$. By Lemma 2.2, we can choose a sequence $\left(\epsilon_{n}\right)_{n=1}^{\infty}$ such that $\epsilon_{n}>0, \epsilon_{n} \rightarrow 0$ and $L_{\epsilon_{n}}=\left\{x \in \Omega:\left(u_{2}-u_{1}\right)(x)<\epsilon_{n}\right\}$ is of finite perimeter. From the divergence theorem (Prop. 2.1) applied on the set $U=L_{\epsilon_{n}} \cap B$ with the vector field $\mathbf{G}(x)=\mathbf{a}\left(x, \nabla u_{2}(x)\right)-\mathbf{a}\left(x, \nabla u_{1}(x)\right)$ and $f=f_{2}-f_{1}$, we obtain

$$
\begin{aligned}
\int_{L_{\epsilon_{n}} \cap B}\left(f_{2}-f_{1}\right) & =-\int_{\partial_{\star}\left(L_{\epsilon_{n}} \cap B\right)}\langle\mathbf{G}, \nu\rangle d \mathcal{H}^{N-1} \\
& =-\int_{\partial_{\star} L_{\epsilon_{n}} \cap B}\langle\mathbf{G}, \nu\rangle d \mathcal{H}^{N-1}-\int_{L_{\epsilon_{n}} \cap \partial B}\langle\mathbf{G}, \nu\rangle d \mathcal{H}^{N-1} .
\end{aligned}
$$

From Proposition 2.3 we get that $\langle\mathbf{G}, \nu\rangle \geq 0$ on $\partial_{\star} L_{\epsilon_{n}} \cap B$. Hence from (3.1) we obtain the following inequality:

$$
\int_{L_{\epsilon_{n}} \cap B}\left(f_{2}-f_{1}\right) \leq-\int_{L_{\epsilon_{n}} \cap \partial B}\langle\mathbf{G}, \nu\rangle d \mathcal{H}^{N-1},
$$

which is equivalent to

$$
\int_{B} \chi_{L_{\epsilon_{n}}}\left(f_{2}-f_{1}\right) \leq-\int_{\partial B} \chi_{L_{\epsilon_{n}}}\langle\mathbf{G}, \nu\rangle d \mathcal{H}^{N-1} .
$$

Now as $\epsilon_{n} \rightarrow 0, \chi_{L_{\epsilon_{n}}} \rightarrow \chi_{z}$ pointwise $\mathcal{H}^{N}$ a.e. in $B$ and pointwise $\mathcal{H}^{N-1}$ a.e. on $\partial B$. Therefore, letting $\epsilon_{n} \rightarrow 0$ in (3.2), we get

$$
\int_{B \cap Z}\left(f_{2}-f_{1}\right) \leq-\int_{\partial B \cap Z}\langle\mathbf{G}, \nu\rangle d \mathcal{H}^{N-1} .
$$

The function $u_{2}-u_{1}$ being nonnegative, it achieves its minimum on $Z$ and since $\partial B \subset \Omega$, we get that $\mathbf{G}(x)=0$ for any $x \in Z \cap \partial B$. Therefore, the right-hand side of (3.3) vanishes and the left-hand side being by assumption nonnegative, we derive

$$
\int_{B \cap Z}\left(f_{2}-f_{1}\right)=0
$$


Since $f_{2}-f_{1} \geq 0$, we conclude that $\mathcal{H}^{N}\left(Z \cap P_{f} \cap B\right)=0$, and hence the theorem is proven.

Clearly, the (SCP) holds iff the set of coincidence $Z$ defined in the above proof is empty. This stronger result can be derived by assuming that the boundary data satisfies $u_{2}-u_{1}>0$ :

Proof of Theorem 1.2. Set $\delta=\inf _{\partial \Omega}\left(u_{2}-u_{1}\right)$, and assume that the conclusion of the theorem does not hold. Then there exists $x_{0} \in \Omega$ with $\left(u_{2}-u_{1}\right)\left(x_{0}\right)<\delta$. Choose $\epsilon$ such that $\left(u_{2}-u_{1}\right)\left(x_{0}\right)<\epsilon<\delta$ and such that $L_{\epsilon}=\left\{x \in \Omega:\left(u_{2}-u_{1}\right)(x)<\right.$ $\epsilon\}$ is of finite perimeter (possible, thanks to Lemma 2.2). Then, $L_{\epsilon}$ is a nonempty open subset that is compactly contained in $\Omega$. Fix a nonempty connected component of $L_{\epsilon}$, which by abuse of notation we denote again by $L_{\epsilon}$. By letting $\mathbf{G}(x)=\mathbf{a}\left(x, \nabla u_{2}(x)\right)-\mathbf{a}\left(x, \nabla u_{1}(x)\right)$, we may appeal to Proposition 2.1 with $U=L_{\epsilon}$, $f=f_{2}-f_{1}$, to obtain

$$
\int_{L_{\epsilon}} f=-\int_{\partial_{\star} L_{\epsilon}}\langle\mathbf{G}, \nu\rangle d \mathcal{H}^{N-1}
$$

From Proposition 2.3 we deduce that $\langle\mathbf{G}, \nu\rangle \geq 0$ on $\partial_{\star} L_{\epsilon}$. Thus, from equation (3.5) we obtain that $f_{1} \equiv f_{2}$ in $L_{\epsilon}$. Since $\partial L_{\epsilon} \cap \partial \Omega=\emptyset$, we get that $u_{2}=u_{1}+\epsilon$ on $\partial L_{\epsilon}$. From Lemma 2.4 we obtain $u_{2} \equiv u_{1}+\epsilon$ in $L_{\epsilon}$, which contradicts the fact that $x_{0} \in L_{\epsilon}$. This proves the theorem.

Remark 3.1. (1) As an easy corollary to the above Theorem we obtain that (SCP) holds in $\Omega$ when no connected component of the coincidence set $\left\{x \in \Omega: u_{1}(x)=\right.$ $\left.u_{2}(x)\right\}$ touches the boundary $\partial \Omega$. Indeed, if this is the case, we can find a sequence of open sets $\Omega_{n} \subset \subset \Omega$ with $\bigcup_{n \in \mathbb{N}} \Omega_{n}=\Omega$ and $u_{2}>u_{1}$ on $\partial \Omega_{n}$, and then we can apply the above Theorem to each $\Omega_{n}$.

(2) In case $\Omega$ and the solutions $u_{1}, u_{2}$ are radially symmetric, it is clear that $L_{\epsilon} \subset \subset \Omega$ for some $\epsilon$, and so the above Theorem applies. An ODE proof was given in this case by [4] and [5].

\section{The Dirichlet case}

In this section, we show the validity of the (SCP) for the Problem $\left(P_{i}\right)$ when the solutions are nonnegative and vanish on the boundary. We also assume that $f_{i} \in L^{\infty}(\Omega), i=1,2$.

From the strong maximum principle arguments of Cuesta and Takáč (see [1, Prop. 2.4), we conclude that in a neighborhood $\mathcal{N}$ of any connected component of $\partial \Omega$, either $u_{1} \equiv u_{2}$ in $\mathcal{N}$ or $u_{2}>u_{1}$ in $\mathcal{N}$. In the latter case, under some more assumptions (see condition $\left(\mathbf{a}^{\alpha}\right)$ in [1]), the Hopf boundary point lemma holds. Therefore, there are two possible cases:

Case $\mathbf{A}: u_{1} \equiv u_{2}$ in a neighborhood of some connected component of $\partial \Omega$;

Case B : $u_{2}>u_{1}$ in a neighborhood of $\partial \Omega$.

Proposition 4.1. Assume $\partial \Omega$ is a $C^{2}$-manifold, and consider $u_{i} \in \mathcal{C}^{1, \theta}(\bar{\Omega})$ to be a solution of

$$
-\operatorname{div}\left(\mathbf{a}\left(x, \nabla u_{i}\right)\right)=f_{i},\left.\quad u_{i}\right|_{\partial \Omega}=0 \quad(i=1,2),
$$

where $f_{i} \in L^{\infty}(\Omega), f_{2} \geq f_{1}$. If case $\mathbf{A}$ occurs, then $u_{1} \equiv u_{2}$ in $\Omega$.

Proof. Let $Z:=\left\{x \in \Omega: u_{1}(x)=u_{2}(x)\right\}$. By hypothesis, $Z \neq \emptyset$. Assume by contradiction that $Z \neq \Omega$. In this case $M:=\max _{\Omega}\left(u_{2}-u_{1}\right)(x)>0$. 
Claim 1. There exists $\epsilon>0$ and a connected component $C$ of $L_{\epsilon}$ such that $\bar{C} \cap \partial \Omega$ coincides with a connected component of $\partial \Omega$ that admits a neighborhood where $u_{1} \equiv u_{2}$.

Since $\partial \Omega$ is a $\mathcal{C}^{2}$-manifold, it has only finitely many connected components. We can thus choose $\rho>0$ small so that for any connected component $\Sigma$ of $\partial \Omega$ either $u_{1} \equiv u_{2}$ in a $\rho$-neighborhood of $\Sigma$ or $u_{2}>u_{1}$ in that neighborhood. Let us define the following sets:

$$
\widetilde{\Omega}=\left\{x \in \Omega: d(x, \partial \Omega)>\frac{\rho}{2}\right\}, \quad \Lambda=\left\{x \in \partial \widetilde{\Omega}: u_{2}>u_{1}\right\},
$$

and let us set

$$
\delta= \begin{cases}\min _{x \in \Lambda}\left(u_{2}-u_{1}\right), & \text { if } \Lambda \neq \emptyset, \\ M, & \text { if } \Lambda=\emptyset .\end{cases}
$$

Now, by Lemma 2.2, the level set $L_{\epsilon}=\left\{x \in \Omega:\left(u_{2}-u_{1}\right)(x)<\epsilon\right\}$ is a set of finite perimeter for $\mathcal{H}^{1}$ a.e. $\epsilon \in(0, \delta)$. We choose and fix a connected component of $L_{\epsilon}$, which by abuse of notation we call again as $L_{\epsilon}$, so that $L_{\epsilon} \cap Z$ is nonempty. Clearly, $L_{\epsilon} \cap \Lambda$ is empty. We can write $\partial L_{\epsilon}=\partial \Omega^{\star} \cup \partial L_{\epsilon}^{\text {int }}$ where $\partial \Omega^{\star}$ is the union of those connected components of $\partial \Omega$ such that $u_{1} \equiv u_{2}$ in a neighborhood of them, and $\partial L_{\epsilon}^{\text {int }} \subset \Omega$.

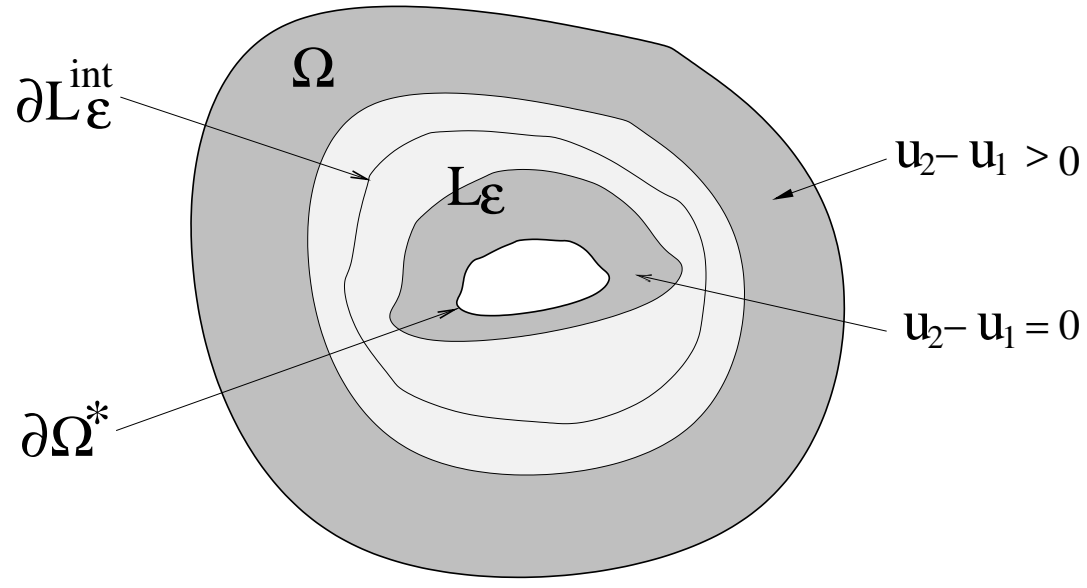

FiguRE 1.

Claim 2. $f_{1} \equiv f_{2}$ in $L_{\epsilon}$.

Let $\mathbf{G}(x)=\mathbf{a}\left(x, \nabla u_{2}(x)\right)-\mathbf{a}\left(x, \nabla u_{1}(x)\right)$. Since $\mathbf{G} \equiv \mathbf{0}$ in a neighborhood of $\partial \Omega^{\star}$ and $\partial L_{\epsilon}^{\text {int }} \subset \subset \Omega$, the divergence theorem (Prop. 2.1) applied with $U=L_{\epsilon}$ gives

$$
0 \leq \int_{L_{\epsilon}}\left(f_{2}-f_{1}\right)=-\int_{\partial_{\star} L_{\epsilon}^{\text {int }}}\langle\mathbf{G}, \nu\rangle d \mathcal{H}^{N-1} .
$$

We also note that $\partial_{\star} L_{\epsilon}^{\text {int }} \subset\left\{x \in \Omega:\left(u_{2}-u_{1}\right)(x)=\epsilon\right\}$. From Proposition 2.3 we have $\langle\mathbf{G}, \nu\rangle \geq 0$ on $\partial_{\star} L_{\epsilon}^{\text {int }}$. Thus, we conclude from (4.1) that $f_{1} \equiv f_{2}$ on $L_{\epsilon}$.

Claim 3. $u_{1} \equiv u_{2}$ on $L_{\epsilon}$. 
Let $w^{\epsilon}=\left(u_{2}-u_{1}\right)-\epsilon$. Then, we note that $w^{\epsilon} \equiv-\epsilon$ in a neighborhood of $\partial \Omega^{\star}$ and $w^{\epsilon}=0$ on $\partial L_{\epsilon}^{\text {int }}$. Noting that $\operatorname{div} \mathbf{G}(x) \equiv 0$ in a neighborhood of $\partial \Omega^{\star}$, we can use $w^{\epsilon}$ as a legitimate test function in the weak formulation to get:

$$
0=\int_{L_{\epsilon}}\left\langle\mathbf{G}(x), \nabla w^{\epsilon}\right\rangle=\int_{L_{\epsilon}}\left\langle\mathbf{a}\left(x, \nabla u_{2}\right)-\mathbf{a}\left(x, \nabla u_{1}\right), \nabla\left(u_{2}-u_{1}\right)\right\rangle .
$$

This immediately implies that $\nabla u_{1} \equiv \nabla u_{2}$ in $L_{\epsilon}$ and hence (since $Z \cap L_{\epsilon} \neq \emptyset$ ), $u_{1} \equiv u_{2}$ in $L_{\epsilon}$. This proves the claim.

From Claim 3, we obtain a contradiction since $u_{1}+\epsilon=u_{2}$ on $\partial L_{\epsilon}^{\mathrm{int}}$ and this contradiction proves Proposition 4.1

The validity of the (SCP) with zero boundary data follows now immediately:

Proof of Theorem 1.3. Since by assumption $f_{1} \not \equiv f_{2}$, Proposition 4.1 implies that Case A never occurs. Therefore, $u_{2}>u_{1}$ in a neighborhood of $\partial \Omega$, and we are reduced to the situation in Theorem 1.2, which concludes the theorem.

\section{ACKNOWLEDGMENTS}

The second author would like to thank the Indo-French Centre (IFCPAR), New Delhi, for sponsoring his visit to the University of Toulouse under project no. 19012. Both authors thank the Department of Mathematics in Toulouse, where this work was done, for the warm hospitality.

\section{REFERENCES}

[1] M. Cuesta and C. P. Takáč, A strong comparison principle for positive solutions of degenerate elliptic equations, Differential Integral Equations 13 (2000), 721-746. MR 2001h:35008

[2] L. C. Evans and R. F. Gariepy, Measure theory and fine properties of functions, Studies in Advanced Mathematics, 1st edition, CRC Press, Boca Raton, FL, 1992. MR 93f:28001

[3] M. Guedda and L. Véron, Quasilinear elliptic equations involving critical Sobolev exponents, Nonlinear Anal. 13 (1989), 879-902. MR 90h:35100

[4] S. Prashanth, Strong comparison principle for radial solutions of quasi-linear equations, J. Math. Anal. Appl. 258 (2001), 366-370. MR 2002a:35010

[5] W. Reichel and W. Walter, Radial solutions of equations and inequalities involving the $p$ Laplacian, J. Inequalities and Applications 1 (1997), 47-71. MR 2000j:34006

[6] P. Tolksdorf, On the Dirichlet problem for quasilinear equations in domains with conical boundary points, Comm. Partial Differential Equations 8(7) (1983), 773-817. MR 85g:35053

Department Mathematics, Hill Center, Rutgers University, 110 Frelinghuysen Rd, Piscataway, NeW Jersey 08854

E-mail address: mlucia@math.rutgers.edu

TIFR Center, IISc. Campus, Post Box No. 1234, Bangalore 560012, India

E-mail address: pras@math.tifrbng.res.in 